\title{
Peak loading and surface pressure fluctuations of a tall model building
}

\author{
Steven J. Daniels, Ian P. Castro, Zheng-Tong Xie* \\ Faculty of Engineering and the Environment, University of Southampton, SO17 1BJ, \\ Southampton, UK
}

\begin{abstract}
Using a recently developed inflow generation approach (Kim et al., 2013), Large-Eddy Simulation (LES) of the flow over the Commonwealth Advisory Aeronautical Council (CAARC) standard tall building has been performed. The surface pressure fluctuations were calculated and are shown to be in reasonable agreement with the measurements in the literature. Numerical experiments were conducted to investigate the effects of the inflow turbulence intensity and integral length scales. Previous research, e.g. Surry and Djakovich (1995) and Huang et al. (2007), suggests a presence of periodicity in the lateral forces on tall buildings, indicating a correlation of the surface pressures on the building's lateral faces. This paper also focuses on the correlations of surface pressure, local wall-normal forces and cross-wind velocity.
\end{abstract}

Keywords: inflow condition; large-eddy simulation; peak pressure; correlation; conditional sampling.

*Email:z.xie@soton.ac.uk; Tel:+44 (0)23 80594493 


\section{Introduction}

At present, a wind engineering toolbox consists of wind tunnel testing of scaled models, field measurements, and mechanical load testing. In recent years, the application of computational fluid dynamics (CFD) has made the numerical evaluation of wind loading an appealing approach. However, despite the popularity of and improvement in computational methods, the problem of providing a fully developed turbulent flow at the inlet, at a reasonable computational expense, is a continuing issue. This is particularly crucial for large-eddy simulation approaches. A common method is to place a subdomain with axially periodic boundary conditions upstream of the model. The sub-domain provides an inflow for the model. This method can achieve a reasonable simulation of a fully developed turbulent flow and deduction of the 'peak loading' on the surface of a bluff body situated in the flow, but can be an expensive process. More recent research has focused on an inlet condition which leads to a more accurate assessment for peak loading and at a cheaper computational expense (e.g. Tamura et al., 2008). The present research applies a recently proposed inflow generator for wind engineering applications (Kim et al., 2013). The generated synthetic inflow turbulence is divergence-free. It is very efficient and has been validated for accurate calculations of pressure fluctuations. The efficacy of this new inflow generator for flows around a tall building was discussed previously in Daniels et al. (2012), in which the effects of inflow turbulence parameters, domain size and mesh resolution were studied.

Flows over isolated square or rectangular buildings have been extensively studied. Analysis has mainly focused on the use of wind tunnel testing but 
has more recently tended towards a numerical approach. One of the more popular high rise building models is the one described by the Commonwealth Advisory Aeronautical Council (CAARC, Moss and Wardlaw, 1970). The CAARC standard tall building model has been extensively used for the analysis of wind loading on tall buildings in wind tunnel studies. Most of these studies use the CAARC building as a means of calibrating experimental techniques. Only more recently has CFD been applied to the CAARC building and most of these studies focus on calibrating various CFD numerical methods. The main source of fluid-structure induced vibrations is thought to be the pressure fluctuations on the building surfaces. Recent research on the CAARC building have focused on the flow patterns and the rootmean-squared (r.m.s.) pressure fluctuation coefficients around the building perimeter (e.g., Dagnew and Bitsuamlak, 2010; Daniels et al., 2012; Huang et al., 2007). Obtaining an accurate estimation for the pressure fluctuations is critical when considering structural vibrations and resulting fatigue concerns. However, this can be challenging. In particular, the approaching flow conditions (e.g. turbulence intensity and integral length scales) and the underlying surface conditions may significantly increase the uncertainties of the prediction.

Our recent work (Daniels et al., 2012) on the numerical analysis of the pressure fluctuations over the CAARC building confirms observations in the literature, e.g. Huang et al. (2007), that the lateral force on the CAARC building exhibits a clear periodicity. This suggests that the surface pressure on one lateral face of the CAARC building must be in some (anti-phase) correlation with that on the opposing side. Using conditional sampling in a 
wind tunnel, Surry and Djakovich (1995) attempted to identify a correlation of pressure peaks on the two lateral faces of a tall building. They found that the pressure peaks at corresponding taps on the opposite lateral faces were in anti-phase (i.e. shifted by 180 degree). This is anticipated because of the antisymmetric nature of vortex shedding. Bartoli and Ricciardelli (2010) investigate the correlations of surface pressure and forces on windward, leeward and two lateral faces of a medium-rise, rectangular plan building in wind tunnel scale, and thus to quantify the errors associated with the quasisteady assumption for wind load evaluation.

To the best of our knowledge, quantitative analysis of the correlations of the pressure fluctuations on the two lateral faces of the CAARC building in atmospheric boundary layer flows have not been reported in the literature. The present study takes account of the effect of various inflow parameters and focuses on a systematic analysis of these correlations, as well as those between local wall normal forces on the lateral faces, and of the cross-wind velocity fluctuations in the wake.

\section{Outline of CFD modelling}

The Large-Eddy Simulation (LES) approaches embedded in the open source CFD code OpenFOAM 1.7.1 (OpenFOAM, 2010) was used throughout this work. The Smagorinsky sub-grid scale (SGS) model with the constant $C_{s}=0.1$ was used. The governing equations and the standard parameters can be found in the literature (e.g. Xie and Castro, 2008). For all simulations the initial durations were sufficiently long (e.g. greater than $500 t^{*}$, where $\left.t^{*}=U_{H} t / D\right)$ to allow the flow to reach full development and the sub- 
sequent averaging durations were about $500 t^{*}$ to obtain converged statistics and were used for further analysis, e.g. conditional sampling.

\subsection{Computational domain and mesh resolution}

The chosen CAARC building for this study is the classical model having a flat-topped prismatic shape, a rectangular cross-section and lateral flat walls with no parapets or other geometric details; the dimensions are: length $(D$, streamwise $)=30 \mathrm{~m}$, width $=45 \mathrm{~m}$, and height $(H$, vertical $)=180 \mathrm{~m}$. The Reynolds number based on the free stream velocity at $y=4 H$ and the height of the building was $R e=3 \times 10^{5}$, which is within the range used in several papers for the validation of wind-tunnel testing. Initially we used a large domain (i.e. $20 H \times 4 H \times 10 H$ ) which is similar as that in Dagnew and Bitsuamlak (2010). A smaller domain (i.e. $10 H \times 4 H \times 8 H$, Fig.1a) was also designed. We noticed that the results obtained using the two domain sizes were consistent, and hence most of the results reported in this paper were obtained from the smaller domain.

Although the CAARC building is a simple rectangular cylinder, the computational mesh generation is not straightforward because of the requirement for a good quality mesh in the near wall regions. Also the total number of cells must be kept relatively low in order to maintain an efficient computation. A structured mesh (Fig.1a) was chosen. The total number of cells used was 7.2 million and 9.4 million for the smaller and larger domains, respectively. The grid stretch ratios of the mesh outward from the CAARC building are as follows: front, 1.08; back, 1.09; sides, 1.06; and top, 1.06.

The $y_{1}^{+}$values are in the range used in the comparative papers of Huang et al. (2007), and Dagnew and Bitsuamlak (2010) (case 1A), with $y_{1}^{+}<10$. 
A wall resolution sensitivity test with $10<y_{1}^{+}<20$ was performed, and is further discussed in Section 3.2. The Smargorinsky SGS model suffers from its excessive dissipation in high shear regions. So the $C_{s}$ constant must be reduced in these regions by using a damping factor, e.g. the Van-Driest damping function (van Driest, 1956),

$$
D=1-\exp \left\{-y^{+} / A^{+}\right\}
$$

where $y^{+}$is the wall normal distance in wall units to the cell centre, and $A^{+}$ is set 26 in OpenFOAM1.7.1. This damping function was applied to the floor of the domain and the surfaces of the CAARC building.

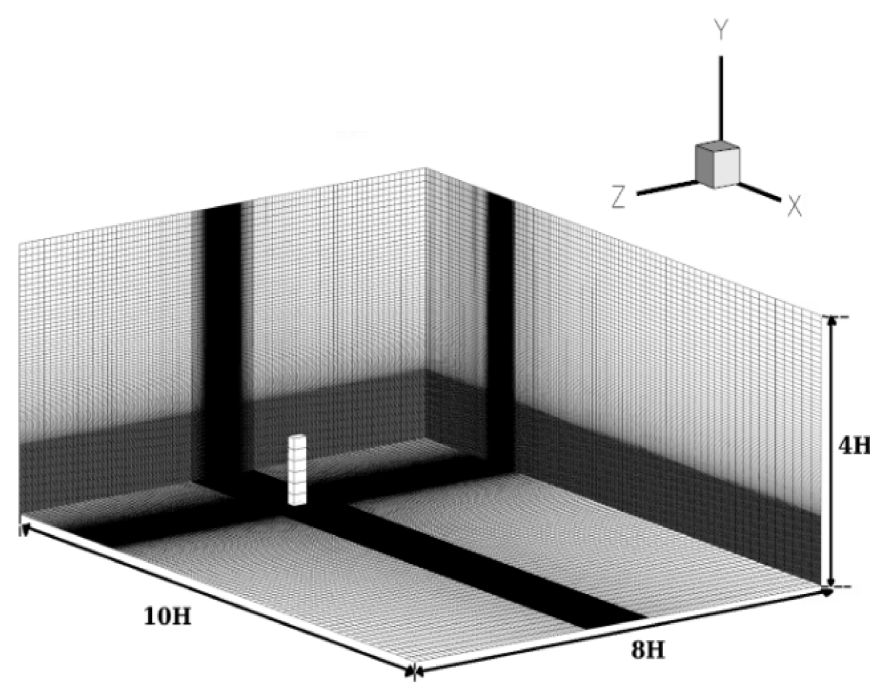

(a)

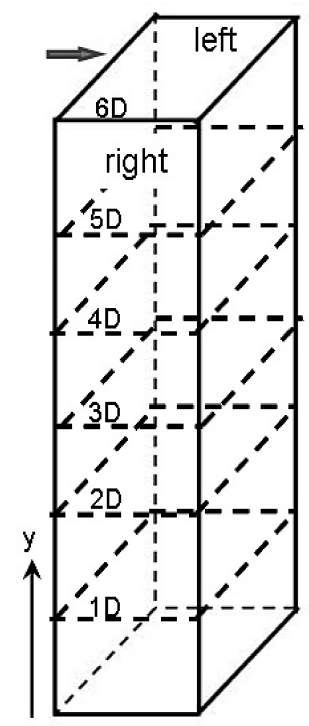

(b)

Figure 1: (a) Overall grid distribution, with coordinates $x, y, z$ corresponding to streamwise, vertical and lateral respectively. (b) A sketch of section partitions on the lateral faces. 


\subsection{Boundary conditions}

The boundary conditions represent the settings used for the wind tunnel test of Dagnew and Bitsuamlak (2010). The lateral sides and top of the domain were set as symmetry-planes. No-slip conditions were used on the walls of the CAARC building and floor of the domain.

A recently proposed inflow generator (Kim et al., 2013) (hereafter XC_DF_07) was used for the inlet condition and is now briefly described. Synthetic turbulence for inflow conditions formulated on a 2-D plane generally produces unphysically large pressure fluctuations in direct numerical and large-eddy simulations (e.g. Xie and Castro, 2008). To reduce such artificial fluctuations a divergence-free inflow generator XC_DF_07 has been developed recently with incompressible flow solvers. Firstly, the generated synthetic turbulence using (Xie and Castro, 2008) is inserted on a transverse plane $\left(x=x_{0}\right)$ in the computational domain near, but crucially not at, the inlet boundary after having solved the momentum equations using the PISO procedure (Issa, 1985). Kim et al. (2013) suggests that XC_DF_07 works well for $x_{0}$ greater than a quarter of the channel depth. In the current simulations, we chose $x_{0}=3 D$. Secondly, these velocities are then adjusted by the velocity-pressure coupling procedure. This means that, on application of the pressure-correction step, the imposed velocities on the plane where the synthetic turbulence is introduced only act as intermediate velocities. Applying synthetic turbulence on the inlet boundary itself, in contrast, fixes those velocities as final velocities throughout one time step. Thirdly, once the synthetic turbulence goes through the velocity-pressure coupling procedure, the velocities are adjusted and satisfy the divergence-free condition. As ex- 
pected, the final velocities are not generally exactly the same as the original. Nevertheless the changes are found to be small. An important feature of this method is that it does not require any additional computational cost.

The inlet condition requires the user to define profiles of the integral length scales (ILS), turbulence intensity (TI), Reynolds stresses and mean velocity. The velocity profile and streamwise velocity fluctuations $\left(\overline{u^{\prime} u^{\prime}}\right)$ were calculated empirically from the mean velocity and turbulence intensity derived from the Dagnew and Bitsuamlak (2010) wind tunnel results. The mean velocity was modelled using a power law exponent of 0.16 with reference velocity $U_{H}=11.7 \mathrm{~m} / \mathrm{s}$ at the building height. The other Reynolds stress components $\left(\overline{v^{\prime} v^{\prime}}, \overline{w^{\prime} w^{\prime}}\right.$, and $\left.\overline{u^{\prime} v^{\prime}}\right)$ were calculated using appropriate relations suggested by Xie and Castro (2009). The streamwise integral length scale of turbulence was deduced from the collected wind tunnel data from Obasaju (1992). And following Xie and Castro (2009), the integral length scales in the streamwise, vertical and lateral directions were set $4 D, D$ and $D$ respectively.

\subsection{Performance of the inflow methods}

Both Huang et al. (2007) and Dagnew and Bitsuamlak (2010) studies used a spectral synthesizer available in ANSYS Fluent to generate the velocity fluctuations (Smirnov et al., 2001). In this method, the fluctuating velocity components are computed by synthesizing a divergence-free velocity vector field from the summation of the Fourier harmonics on the basis of the input turbulence boundary conditions. However, this method leads to a rapid energy decay in the inertial-subrange of turbulence, creating an early cut-off frequency as detailed in Huang et al. (2010). 

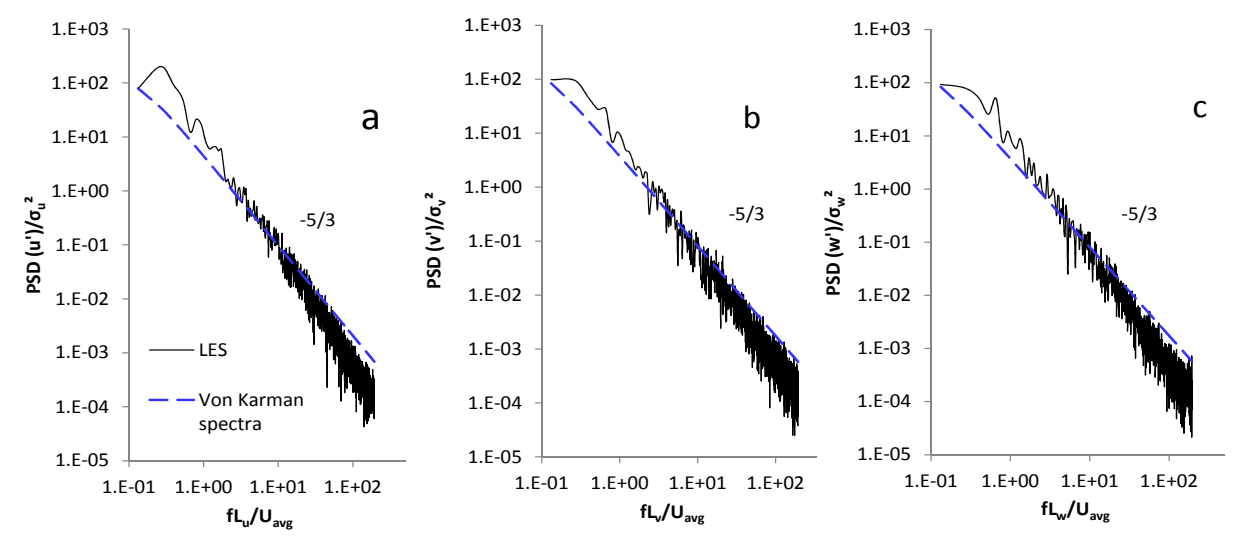

Figure 2: Power spectral density of velocity fluctuations at $x=3.17 D, y=D$ on the central plane. (a) $u^{\prime}$, (b) $v^{\prime}$, (c) $w^{\prime}$.

In order to improve this, Huang et al. (2010) apply a Dirac delta function to the Smirnov et al. (2001) method to construct velocity spectra with appropriate shapes. The Huang et al. (2010) method pays a high price on CPU time for the turbulence generation. With the default settings in Huang et al. (2010), it is 250 times more expensive than the Smirnov et al. (2001) method. Huang et al. (2010) comment that this method is, however, more suitable than the earlier ones for parallel computations, which are naturally much more efficient.

It is to be noted that the major feature of the Xie and Castro (2008) method is its very high efficiency and its flexibility. Its improved version the new divergence-free method (Kim et al., 2013) - has a similar efficiency. The velocity spectra presented in Huang et al. (2010) show a substantial improvement compared with those using the Smirnov et al. (2001) method. Our 
methods (Xie and Castro, 2008; Kim et al., 2013) show a similar performance as the Huang et al. (2010) method in this respect. Fig.2 shows a power spectral density of velocity fluctuations at a station (i.e. at $x=3.17 D, y=D$ on the central plane) immediately downstream of the $x_{0}$ plane where the synthetic turbulence is imposed. These are consistent with those in Xie and Castro (2008) and Kim et al. (2013). The von Karman wind spectra (ESDU85020, 2001) are also included in Fig.2 for comparison. In this model, the spectra for the three velocity components are described,

$$
\begin{gathered}
\frac{P S D\left(u^{\prime}\right)}{\sigma_{u}^{2}}=\frac{4 n_{u}}{f\left(1+70.8 n_{u}{ }^{2}\right)^{5 / 6}} ; n_{u}=f L_{u} / U_{\text {avg }} \\
\frac{P S D\left(\xi^{\prime}\right)}{\sigma_{\xi}^{2}}=\frac{4 n_{\xi}\left(1+755.2 n_{\xi}^{2}\right)}{f\left(1+283.2 n_{\xi}^{2}\right)^{11 / 6}} ; n_{\xi}=f L_{\xi} / U_{\text {avg }} ; \quad \xi=v \text { or } w
\end{gathered}
$$

where $L$ is the integral length scale, $f$ is the frequency, $\sigma^{2}$ is the variance, and $U_{\text {avg }}$ is the local average velocity. The LES spectra show an evident slope $-5 / 3$. However, at very high frequencies the LES spectra show a slightly steeper slope, which might be due to the limited resolution.

It is crucial to check the flow development from the inlet to immediately upstream of the CAARC building, which might be affected by the coarse resolution in the near inlet region. We extensively checked the mean and turbulence intensity profiles. We found that the turbulence flow converged very quickly, e.g. from $3 D$ downstream of $x_{0}$ - where the inflow synthetic turbulence was inserted. 


\section{Data analysis and discussion}

\subsection{Mean and r.m.s. fluctuating pressure}

While there is a wide range of wind tunnel tests which might be used to validate our results, such tests have to be chosen carefully. Early experimental testing, such as the data collected from five institutions by Melbourne (1980), generally shows some inconsistencies between the pressure measurements. Huang et al. (2007) made a detailed comparison of the Melbourne (1980) data and deduced that the effects of the inflow conditions on the pressure results is significant. Therefore for the purpose of validation for LES, we choose wind tunnel data (Huang and Gu, 2005; Dagnew and Bitsuamlak, 2010) having similar inlet characteristics to those employed herein.

The mean pressure and r.m.s. of pressure fluctuation coefficients are respectively defined as $C_{p}=\left(p_{m}-p_{r e f}\right) /\left(0.5 \rho U_{H}^{2}\right)$ and $c_{p r m s}^{\prime}=\left(p_{r m s}\right) / 0.5 \rho U_{H}^{2}$, where $U_{H}$ is the undisturbed velocity at the CAARC building height at the inlet, $p_{\text {ref }}$ is the mean pressure taken at $3 \mathrm{H}$ above and $\mathrm{H}$ upstream of the CAARC building, $p_{r m s}$ is the r.m.s. of the pressure fluctuations and $\rho$ is the air density. A Large-Eddy Simulation (LES) in Huang et al. (2007) with the inflow method from Smirnov et al. (2001), a Reynolds Averaging NavierStokes (RANS) RNG k- $\epsilon$ calculation (Dagnew and Bitsuamlak, 2010), and wind tunnel tests (Dagnew and Bitsuamlak, 2010; Huang and Gu, 2005) were all used to evaluate the mean pressure and root-mean squared (r.m.s.) surface pressure fluctuations; results are compared with the present computations (using XC_DF_07). The mean and r.m.s. fluctuating pressure on the CAARC building are plotted in Figs. 3 and 4 respectively. These include the pressure results for cases in which the inlet turbulence intensity was altered 
by either doubling or reducing to one hundredth the base value, in these cases the integral length scales $\left(L_{u}, L_{v}\right.$, and $\left.L_{w}\right)$ remained the same (base). As well as this, the effect of the integral length scales were studied by halving the base values while maintaining the same (base) turbulence intensity. In the case of the RANS calculation, the averaging technique failed to capture the separation bubble on the corners of the CAARC building; hence the large underestimation of mean pressure at $x / D=1.5$ in Fig.3. The present LESpredicted mean pressure coefficient over the building perimeter at $y=2 / 3 H$ is in good agreement with all of the results, apart from the RANS data.

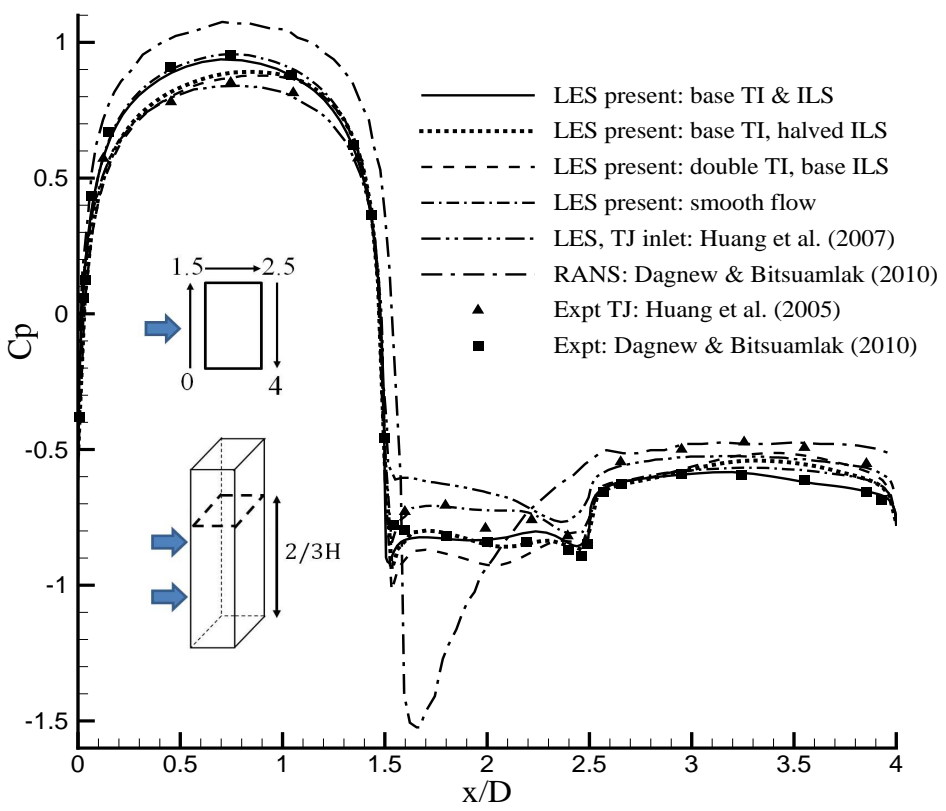

Figure 3: Mean pressure coefficients over the CAARC building perimeter $(y=2 / 3 \mathrm{H})$.

For the r.m.s. pressure fluctuations (Fig.4), the XC_DF_07 inlet condition captures the high fluctuations on the rear corners of the CAARC building but shows some discrepancies in the magnitude of the fluctuations on the 


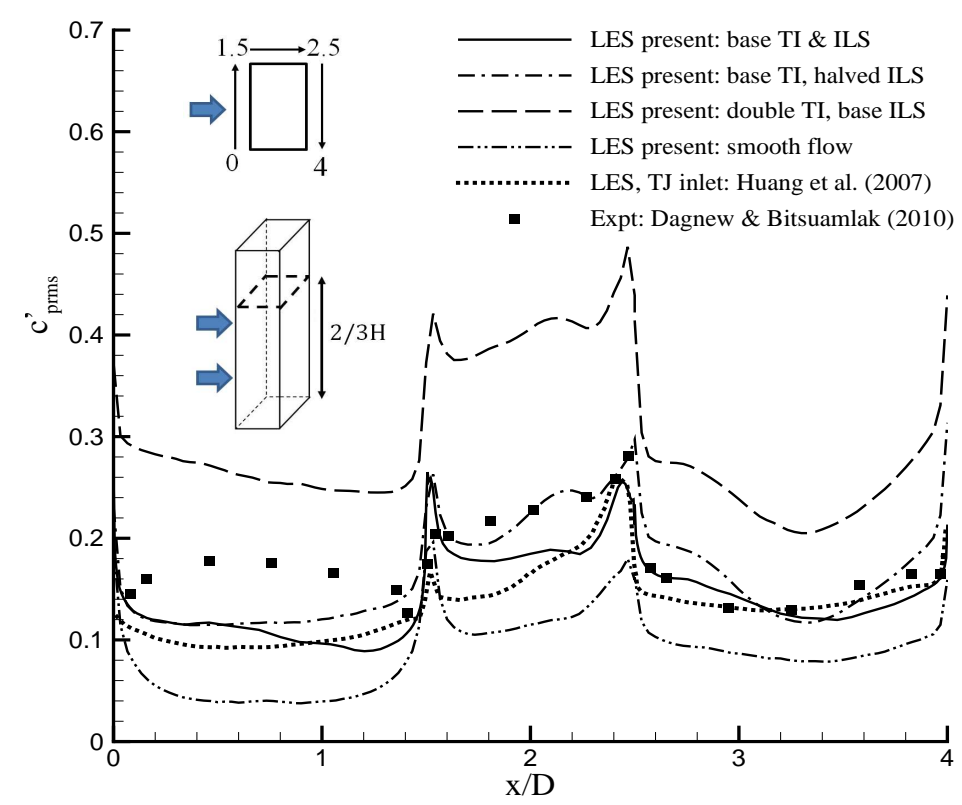

Figure 4: Root-mean-squared pressure fluctuations around the perimeter of the CAARC building at $y=2 / 3 H$.

lateral sides. Such discrepancies could be due to differences in the specified mean velocity power-law exponent, and turbulence intensity at the inlet. Huang et al. (2007) suggested that the pressure fluctuations on the CAARC building are less sensitive to the velocity profile than the turbulence intensity, but the mean pressure is more sensitive to the mean velocity profile than the turbulence intensity. Such observations agree with this present study. Although the mean velocity and turbulence intensity for the inlet condition were taken from Dagnew and Bitsuamlak (2010), the approximations for the Reynolds stress components are slightly different, which could explain the differences in the r.m.s. surface pressure fluctuations. The mean pressure around the CAARC building is in agreement with Dagnew and Bitsuamlak (2010)'s wind tunnel test, suggesting that the mean velocity profile has the 
dominant effect on the mean pressure.

The LES results in Fig. 4 generally agree with the wind tunnel data. However there is a slight contradiction concerning where the pressure fluctuations on the front face are at a maximum, and there is debate about this in previous studies of the CAARC building. Goliger and Milford (1988) suggested that the wide range of turbulence intensities and experimental errors presented by many wind tunnel tests may be the reason for the variations in the pressure fluctuations on the front face of the CAARC building. Nevertheless, the distribution of fluctuating pressure on a square-section cylinder (Bearman and Obasaju, 1982), which has the lowest pressure fluctuation at the centre of the windward face, provides some evidence to support the present results.

Research on the effects of integral scale on the pressure fluctuations over bluff bodies have dealt almost exclusively with low-rise buildings (e.g. Hold $\varnothing$ et al. (1982)). Xie and Castro (2008) investigated numerically the effects of the integral length scales of the flow over an array of staggered cubes of uniform height. Their results show that the integral length scale has a small effect on the flow statistics (i.e. the Reynolds stresses), as long as the estimated integral length scales are within an appropriate range. No surface pressure fluctuations were reported in Xie and Castro (2008). Figs. 3 and 4 show the changes of the pressure results by halving the base integral length scales. The effect of the integral length scales are small for both mean and r.m.s. fluctuating pressures, especially when compared with the effect due to the variation of the turbulence intensity in Fig.4. The small variation of $C_{p}$ and $c_{p r m s}^{\prime}$ due to the changes of the integral length scales in Figs.3 and 4 
seems within the numerical uncertainties, e.g. averaging error. This confirms the point in Xie and Castro (2008) and also suggests that the integral length scale has a small effect on the surface pressure fluctuations as long as they are within an appropriate range.

\subsection{Correlations between aerodynamic forces and surface pressure fluctua-} tions

Each lateral face of the CAARC building was partitioned into 6 sections in the vertical direction (Fig.1b). Pressure fluctuation coefficients at two stations of each section were sampled. The total wall-normal force on each section was also sampled.

Surface pressure fluctuations on the two sections at $y=3 D$ of the two lateral faces are clearly in anti-phase (i.e. shifted by 180 degree), as shown in Figs.5a and b. The total wall-normal force fluctuations on the left lateral section at $y=2.5 D$ (Fig.5c) are also in anti-phase with those on the right lateral section shown in Fig.5d. The lateral velocity fluctuations in the wake at various distances from the leeward face of the building (Figs.5e and f) are almost in phase. Figs.5a-f collectively show the same dominant frequency, with the Strouhal number $f D / U_{H} \sim 0.076$, where $f$ is the frequency, $D$ is the building length in streamwise direction and $U_{H}$ is the reference velocity which is the inlet velocity at building height. This is consistent with the dominant frequency of the total lateral force on the CAARC building (Daniels et al., 2012), and is also in agreement with the literature, e.g. Huang et al. (2007). These again confirm that the vortex shedding from the lateral faces is the genuine mechanism (Surry and Djakovich, 1995) driving the oscillation of the aerodynamic forces. 

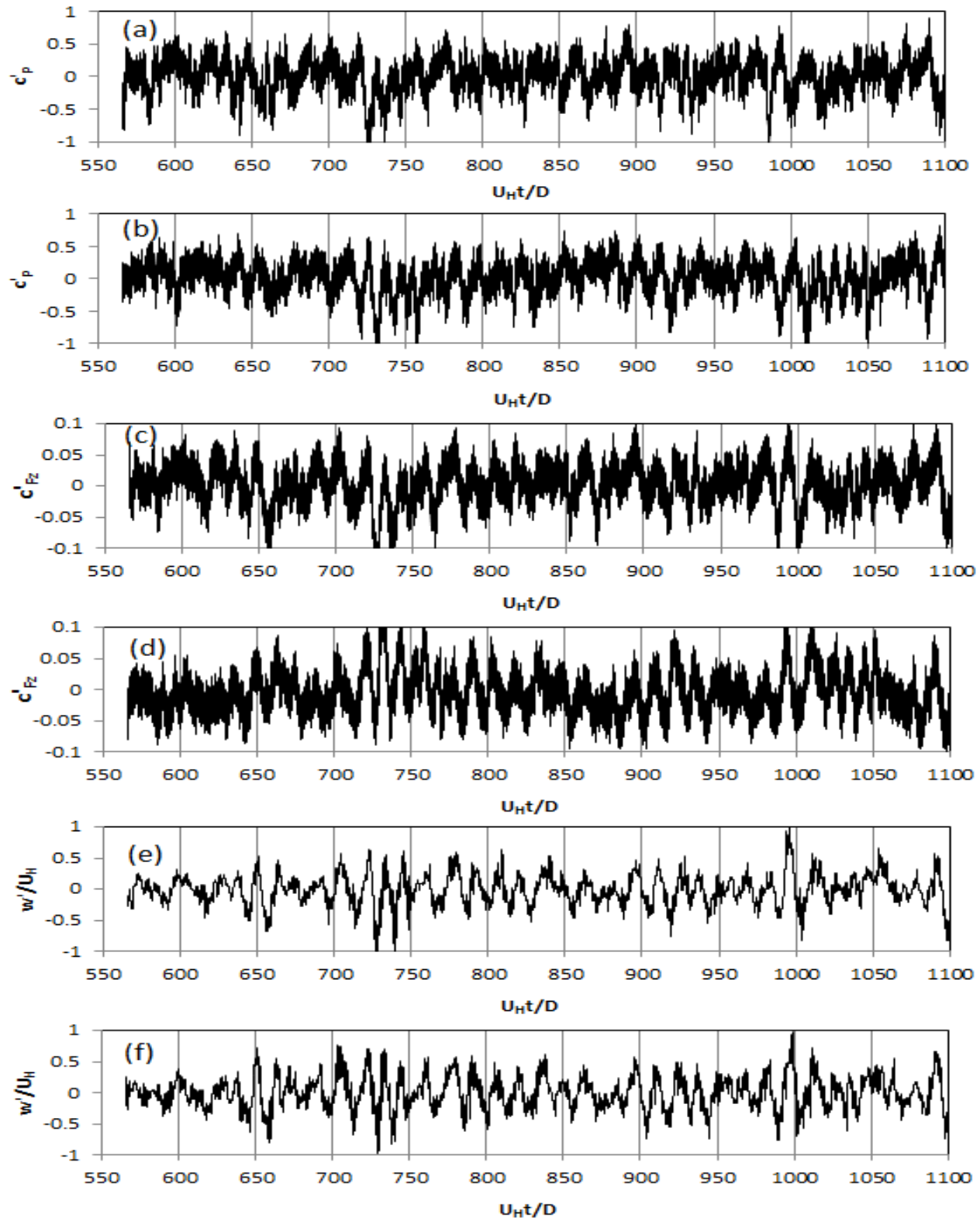

Figure 5: (a) A time series of the pressure coefficients on left lateral face at $y=3 D$ at the centre of the section, (b) as for (a) but on the right lateral face; (c) a time series of the coefficient of the wall-normal force fluctuation on the left lateral face section at height $y=2.5 D,(\mathrm{~d})$ as for (c) but on the right lateral side; (e) a time series of the dimensionless spanwise velocity fluctuation $w^{\prime}$ at $0.17 D$ from the leeward side and at $y=3 D$ on the central plane, (f) as for (e) but at $0.5 D$ from the leeward side. 

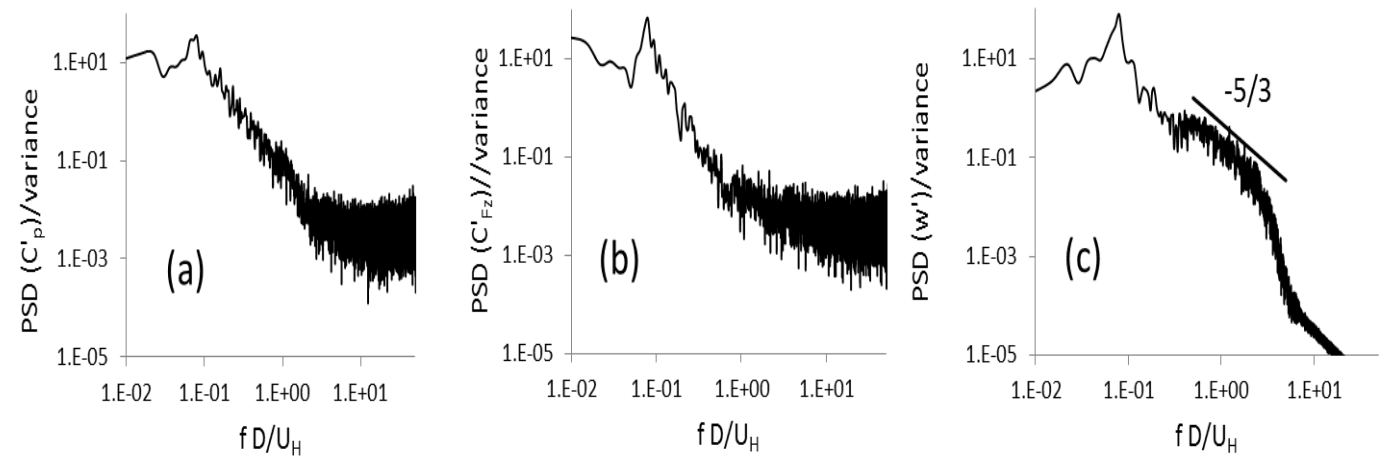

Figure 6: Power spectral density of (a) pressure fluctuation coefficient on the left lateral face at $y=3 D$, and at a distance $0.5 D$ from the leading corner (Fig.5a), (b) wall-normal force fluctuation coefficient on the left lateral face section at height $y=2.5 D$ (Fig.5c), and (c) spanwise velocity fluctuations at $y=3 D$ and at a distance $0.5 D$ from the leeward side on the central plane (Fig.5f).

The total wall-normal forces on sections at $y=2.5 \mathrm{D}$ were highly correlated (i.e. correlation coefficients exceeding 0.9) with the surface pressures on the same section. This is clearly shown in Figs.5a and c. This might not be surprising because the total wall normal force is the integration of the surface pressure. The wake velocities in Figs.5e and $\mathrm{f}$ also show a high correlation with the surface pressures (compare Figs.5a and b with Figs.5e and f). Also a phase lag between the wake velocities and the surface pressures is evident. This will be further discussed in $§ 3.3$.

Fig.6a shows the power spectral density of the pressure fluctuation coefficient on the left lateral face at $y=3 D$, and at a distance $0.5 D$ from the leading corner (the corresponding time series is Fig.5a). The spectra shows an evident peak at $f D / U_{H} \sim 0.076$, confirming the dominant vortex shedding frequency stated earlier. The spectra of the time series in Fig.5b sampled 

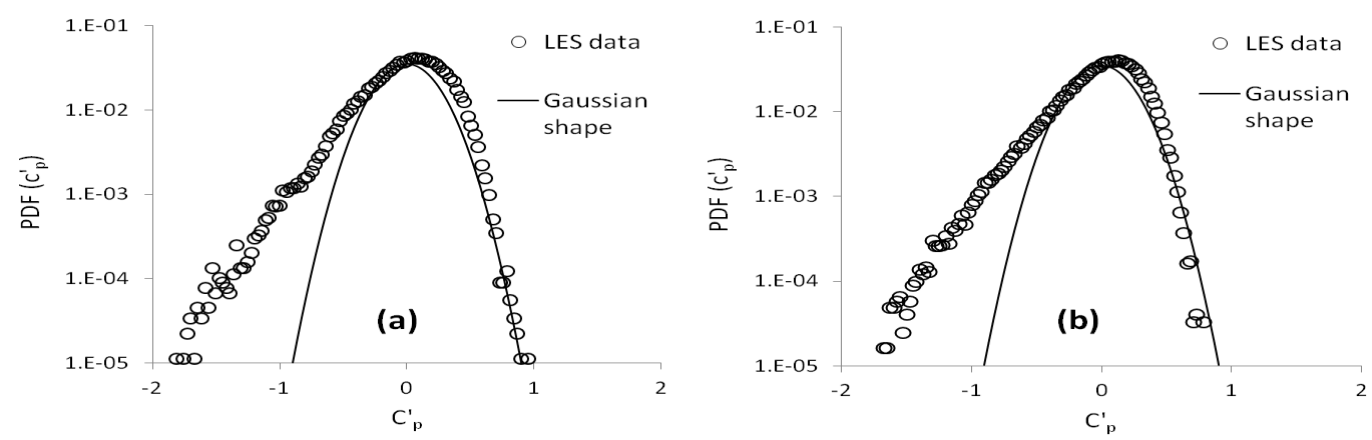

Figure 7: Probability Density Functions (PDFs) of surface pressure fluctuation coefficient on the left lateral face at a distance $0.5 D$ from the leading corner.(a) at $y=3 D$ (Fig.5a), (b) at $y=4 D$.

at the right lateral face is almost identical as that at the left lateral face in Fig.6a. Fig.6b shows the power spectral density of the wall-normal force fluctuation coefficient (the time series is Fig.5c) on the left lateral face section at height $y=2.5 D$, which also shows a dominant frequency $\left(f D / U_{H} \sim\right.$ 0.076). This is expected as the section wall-normal force is highly correlated with the local surface pressure. Fig.6c shows the power spectral density of the spanwise velocity fluctuations (Fig.5f) at $y=3 D$ and at a distance $0.5 D$ from the leeward side on the central plane. It again confirms the dominant frequency $\left(f D / U_{H} \sim 0.076\right)$ in the wake. An inertial sub-region is evident in Fig.6c in the wake region.

It has been reported in the literature (eg. Gioffre et al., 2001) that the Probability Density Function (PDF) of surface pressure fluctuations is nonGaussian. The present numerically predicted surface pressure fluctuations on the lateral and the leeward faces are likewise non-Gaussian; Fig.7 shows two examples. Fig.7a shows a PDF of surface pressure fluctuation coefficient (see 


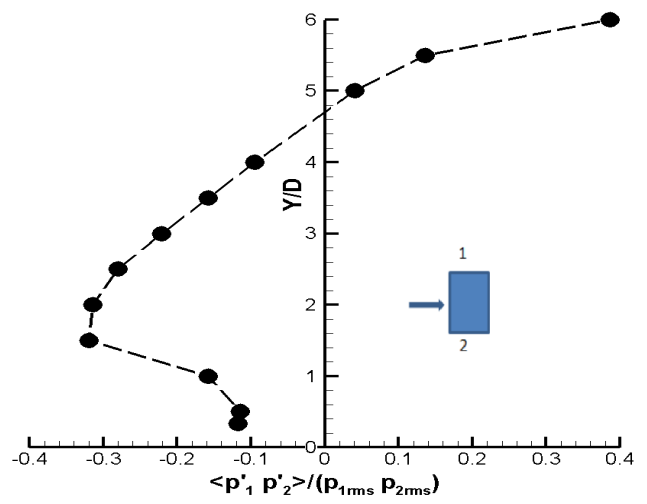

(a) Correlations between the corresponding surface pressures at $0.5 D$ from the leading corner on the two lateral faces.

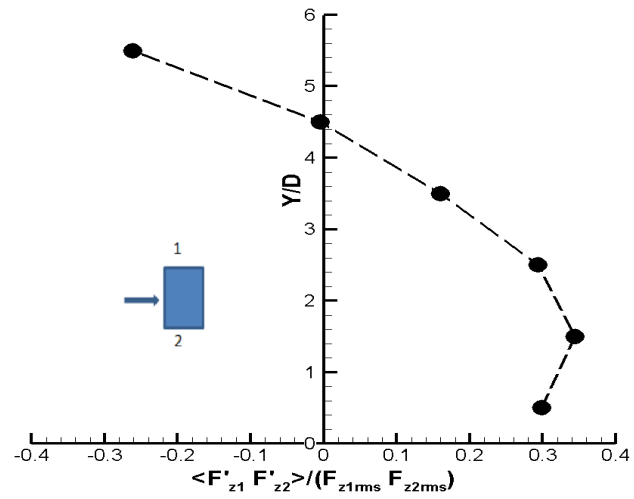

(b) Correlations between the corresponding total wall-normal forces of the two lateral face sections (see Fig.1b).

Figure 8: Correlations of the pressures and wall-normal forces on the lateral faces.

the time series in Fig.5a) on the left lateral face at a distance 0.5D from the leading corner and at $y=3 D$. The LES data show a much longer negative tail in the PDF than that of a Gaussian distribution. Fig.7b shows a PDF of the pressure fluctuation at $y=4 D$ on the same lateral face; this is quite similar to that in Fig.7a.

Fig.8a shows the correlations between the corresponding surface pressure fluctuations at $x=0.5 D$ from the leading corner on the two lateral faces, and Fig.8b shows the correlations between the corresponding total wall-normal force fluctuations of the two lateral face sections (see Fig.1 b), where subscripts 1 and 2 refer to left and right lateral faces respectively. Figs.8a and 8b show similar but oppositely signed correlation. The maximum of the absolute values of the correlations at the medium height is only around 0.3 . This is certainly because the high inflow turbulence intensity reduces such correlations. Note that it is not possible to distinguish between a change in 
coherence of the fluctuations and a change in phase using simple correlations only at zero lag. Instead, we address the issue of phase using conditional sampling in $§ 3.3$.

The correlation in Fig.8a increases to zero at $y \sim 4.5 \mathrm{D}$ and then to nearly +0.4 at the top of building. This is because large structures shedding from the top face generate in-phase pressure fluctuations on the top face and the two lateral faces near the top. We also checked time series of pressure fluctuations on the windward and leeward faces near the top. They all had a clear in-phase tendency with the pressure fluctuations on the two lateral faces near the top. (There might be other mechanisms to be investigated, but we have not pursued this further.) Again it is not possible to distinguish between a change in coherence of the fluctuations and a change in phase using such simple correlations. Near the ground, the correlations both seem to approach zero. This might be due to the very small scale eddies in the near surface region. The effect of the first wall grid resolution was also investigated. We changed the average first grid resolution from 20 wall units to 10 wall units, but found no significant effects.

3.3. Deductions from conditional sampling: peak surface pressure, aerodynamic forces and wake velocities

Conditional sampling and averaging has been widely used for quantitative analysis in various problems, e.g. identifying coherent structures in quasi-periodic and periodic flows (Antonia, 1981) and determining peak surface pressure fluctuations (Surry and Djakovich, 1995; Lam and Zhao, 2002). Usually a number of simultaneous time histories of various variables at various stations are considered. Only one time history of one variable at one 
station is chosen as the triggering signal. If the value of this variable at a certain time (i.e. triggering time) exceeds the threshold, then all of the simultaneous time histories before and after the triggering time within a small constant duration are sampled. This corresponds to one event. The triggering time for every event is translated to zero. Finally all of the time histories of the events are averaged. This technique significantly reduces the size of the massive dataset and identifies the extreme events more clearly.

In the context of wind loading problems the technique is particularly useful for investigating the correlation of the peak pressures at different locations on the building surfaces, as well as the correlation of the peak pressure and the velocity components. It is also very useful for studying the correlation of the peak surface pressure and the peak loading on the buildings. We present here our specific technique and some of the results.

As in $§ 3.2$, each lateral face of the building was evenly partitioned into 6 sections in the vertical direction (Fig.1b). Surface pressure fluctuation coefficients at stations on the lateral faces, with heights $y=5 D, 4 D, 3 D$, $2 D$ and $1 D$ respectively, and a distance $0.5 D$ from the windward face of the building, were recorded for every time step for a duration $426 t^{*}$ (i.e. 1162s), where $t^{*}=U_{H} t / D$. The wall normal force coefficients on all the sections were recorded simultaneously for the same duration. Lateral velocity components at stations on the central plane in the wake region were also recorded simultaneously.

The pressure fluctuation coefficient $c_{p}^{\prime}=\left(p-p_{m}\right) /\left(0.5 \rho U_{H}^{2}\right)$ at $y=3 D$ and a distance $0.5 \mathrm{D}$ from the leading corner on the left lateral face (Fig.1b) was chosen as the triggering signal. Alternatively, it would be straightforward 
to use the local force as the trigger signal (Lam and Zhao, 2002). In order to get sufficient number of events for averaging, the triggering threshold was set to be -0.53 . We focused more on the negative peak $c_{p}^{\prime}$ because it was of greatest interest (Surry and Djakovich, 1995). Using the conditional sampling technique, the total number of sampled events $n$ was 21 . It is to be noted that the period of the vortex shedding was about $13 t^{*}$ (i.e. $36 \mathrm{~s}$ ).

The conditionally averaged time histories of the pressure fluctuation coefficients at heights $y=5 D, 4 D, 3 D, 2 D$ and $1 D$ on the both lateral faces are shown in Fig. 9. The peak $c_{p}^{\prime}$ shows an evident anti-phase correlation with that at the same height on the opposing lateral face. This was also observed by Surry and Djakovich (1995), whereas on the same lateral face all of the peaks at various height were in an excellent correlation. Surry and Djakovich (1995) also observed that the peaks first occurred at upper levels then sequentially towards the bottom of the building. Fig.9 shows that the high peaks at $y=D$ occur approximately $2 t^{*}$ (i.e. 6s) later than those at $y=5 D$.

Fig.10 (a) shows the sum of the conditionally averaged time histories of the wall-normal force coefficients on the two opposite lateral face sections. These are the lift coefficients $C_{l}=L /\left(0.5 \rho U_{H}^{2} A\right)$, where $L$ is the total lateral forces on the two lateral sections at the same height, $\rho$ is the air density, $U_{H}$ is the reference velocity and $A$ is the surface area. Fig.10(a) shows the same pattern as that in Fig.9. The lift coefficients at all heights are highly correlated. The high peak at $y=0.5 D$ occurs approximately $2 t^{*}$ (i.e. 6s) later than those at $y=5.5 \mathrm{D}$. This is consistent with the same observation shown in Fig.9. Fig.10(a) also shows that the peaks of lift coefficients are 

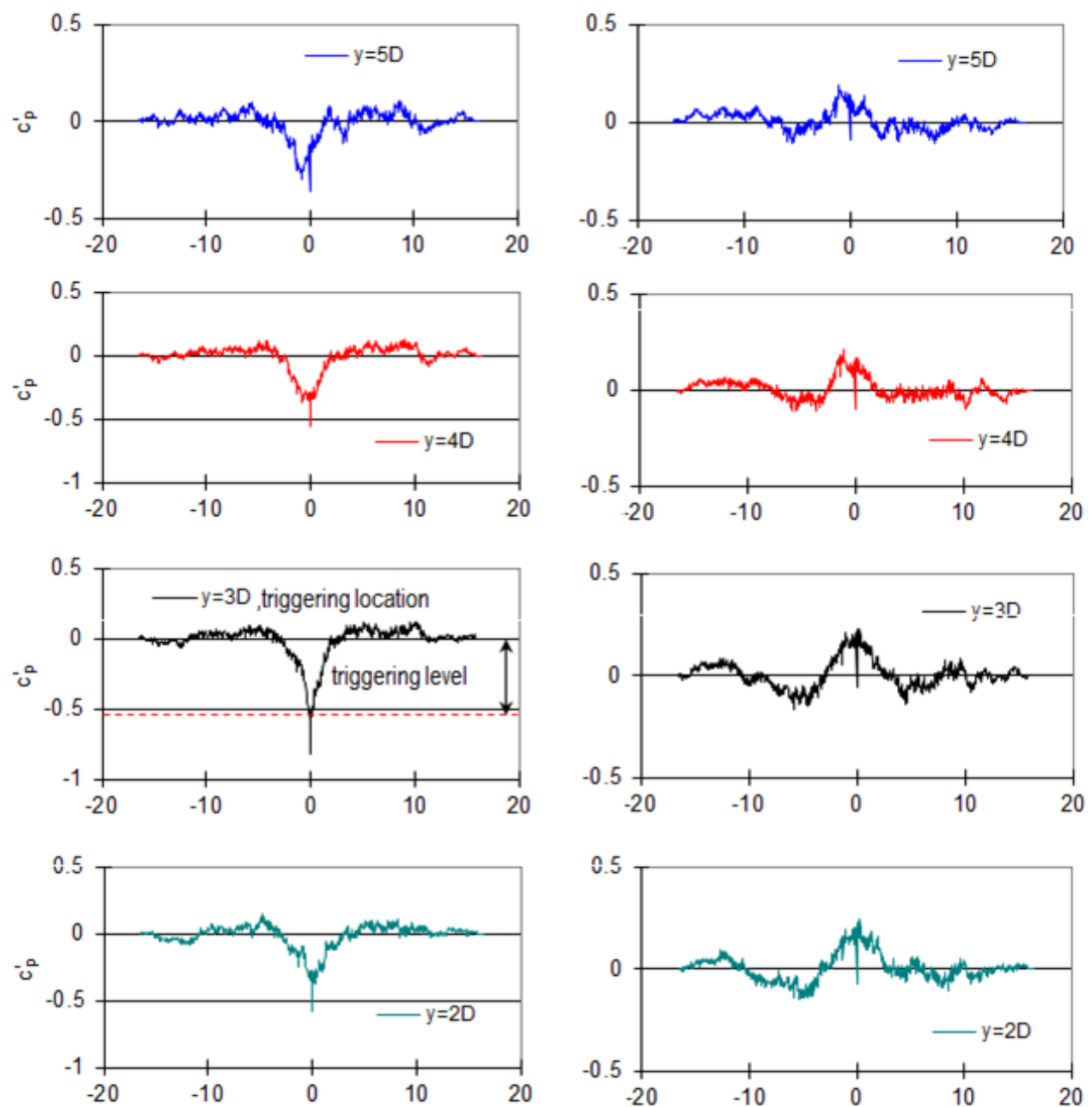

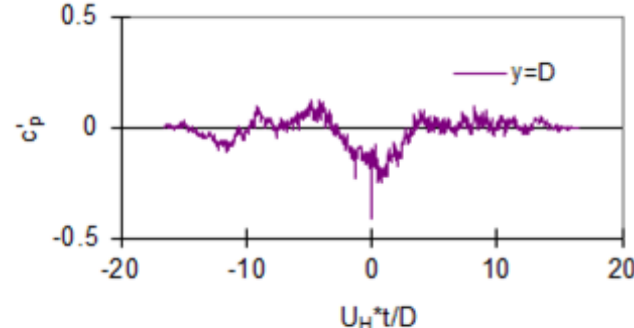

(a) Left lateral face

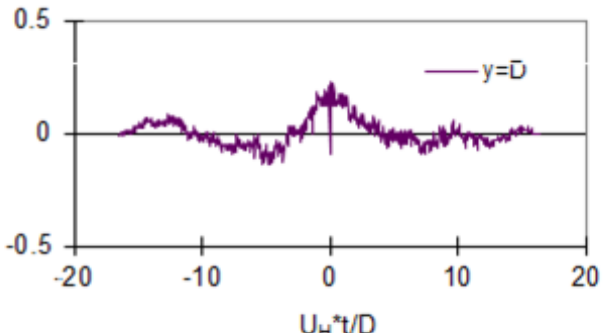

(b) Right lateral face

Figure 9: Conditionally averaged $(\mathrm{n}=21)$ time series of pressure fluctuation coefficients at heights $y=5 D, 4 D, 3 D, 2 D$ and $1 D$. (a) Left lateral face. (b) Right lateral face. 

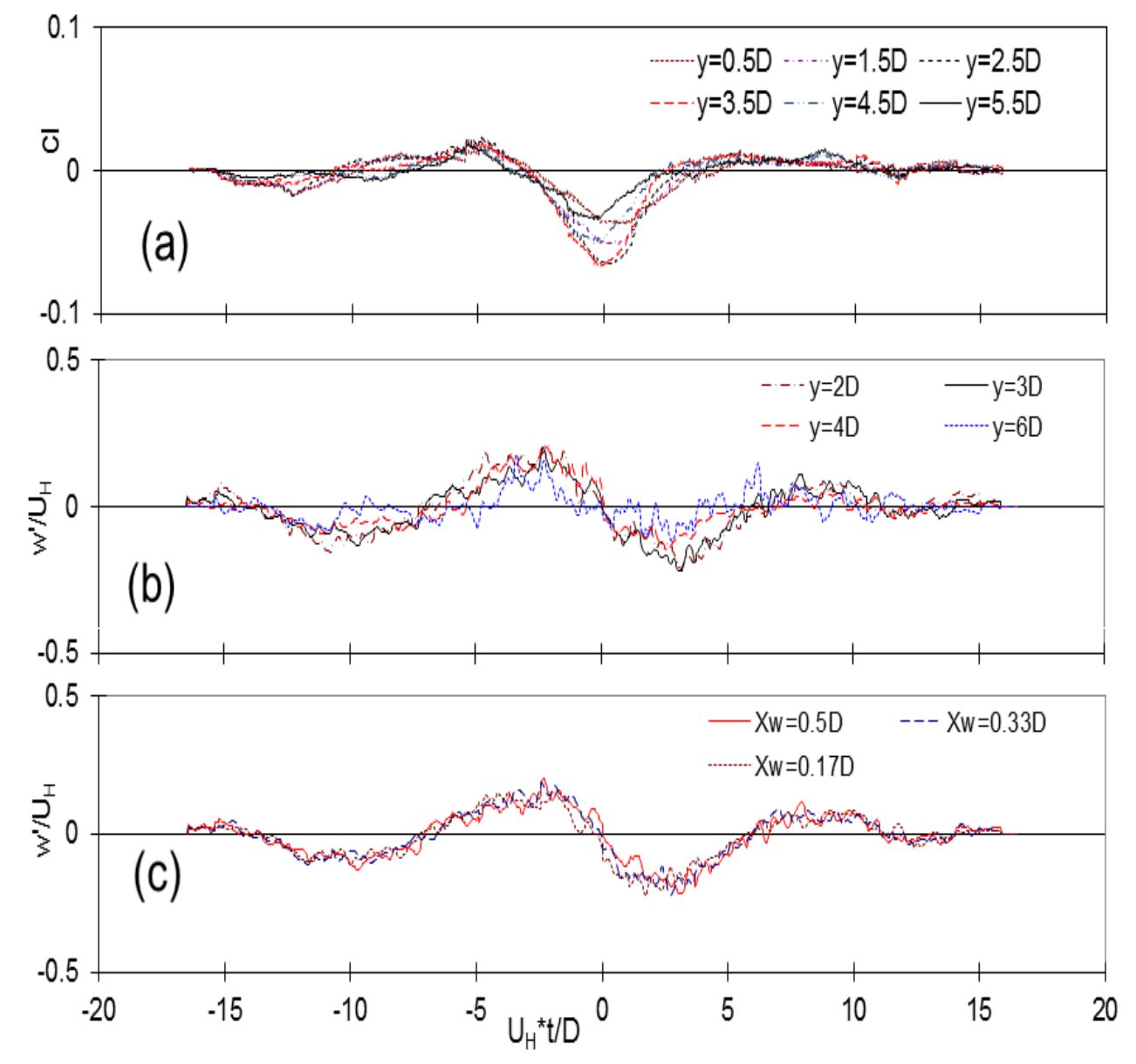

Figure 10: Conditionally averaged $(\mathrm{n}=21)$ time series. (a) Lift coefficients at heights $y=5.5 D, 4.5 D, 3.5 D, 2.5 D, 1.5 D$ and $0.5 D$. (b) Lateral velocity in the wake; distance to the leeward face $x_{w}=0.5 D ; y=6 D, 4 D, 3 D$ and $2 D$. (c) Lateral velocity in the wake; distance to the leeward face $x_{w}=0.5 D, 0.33 D$ and $0.17 D ; y=3 D$.

in excellent correlation with the peaks of pressure fluctuations at the same heights on the left lateral face shown in Fig.9.

Fig.10(b) plots conditionally averaged time histories of lateral velocity fluctuations in the wake region at a distance from the leeward face $x_{w}=0.5 D$ and at four heights $y=6 D, 4 D, 3 D$ and 2D. Fig.10(c) plots averaged time histories at $x_{w}=0.5 D, 0.33 D, 0.17 D$ and $y=3 D$. All of the time histories 
at various stations are closely in-phase. Nevertheless, the pattern of the high peaks in Fig.10 (b,c) is different from that in Figs.9 and 10(a). Fig.10 (b,c) shows that $w^{\prime}$ reduces to zero at $t^{*}=0$. Note that the extreme events of the surface pressures and lift coefficients occur at around the triggering time $t^{*}=0$ and that the mean lateral velocity $w_{m}=0$. The negative peak of $w^{\prime}$ occurs at about $t^{*}=3$, which is approximately at a phase angle $90^{\circ}$, taking $t^{*}=0$ as the time for phase angle $0^{\circ}$.

Surry and Djakovich (1995) placed monitors at various distances from the windward side of the building to sample the lateral velocity. They observed a time delay between the peak velocity and the peak surface pressure on the building lateral faces. They commented that this delay was consistent with a convected disturbance traveling at a certain speed. They also speculated that the peak velocity was associated with the previous vortex shedding from the opposite leading corner. Similarly, the time delay between the negative peak surface pressure and the negative peak lateral velocity in the wake is probably associated with the current vortex shedding from the leading edge of the left lateral face (see Fig.11a-c) and travelling to the near-leeward face region.

Fig.11 shows typical instantaneous velocity vectors and contours of pressure fluctuation coefficients at phases $-90^{\circ}, 0^{\circ}$ and $90^{\circ}$, again assuming that $t^{*}=0$ is corresponding to phase angle $0^{\circ}$. While attached to the left lateral faces (Fig.11b), the vortex enhances the velocity magnitude at the outside edge of the separation bubble, and subsequently produces a negative peak surface pressure fluctuation. When the surface pressure exceeds the prescribed threshold, the conditional sampling is triggered. The dimensionless 

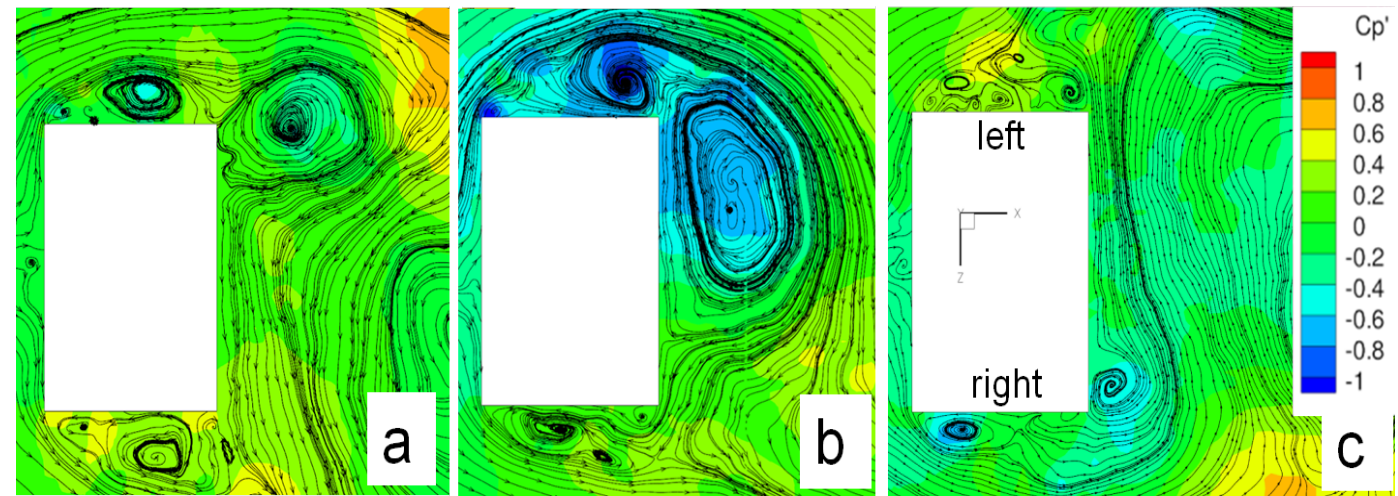

Figure 11: Typical instantaneous velocity vectors and contours of pressure fluctuation coefficients at $y=3 D$. (a) at phase $-90^{\circ}$. (b) at phase $0^{\circ}$. (c) at phase $90^{\circ}$.

apparent travel time is consistent with the time delay $t^{*}=2.5$, given that the travelling velocity is less than $U_{H}$ and the travelling distance is about $2 D$, which is the distance from the centre of vortex at the left lateral face in Fig.11b to the centre (not shown due to space) of vortex in the wake in Fig.11c. Figs.11a, b and c respectively show a positive peak, a very small value and a negative peak of the $w$ velocity in the near-leeward-face region on the central plane. The surface pressures on both lateral sides in Figs.11a and 11c are small and of the same order (also see Fig.9). These results also confirm that the surface pressures are correlated to the wake velocities, as we noticed in $\S 3.2$.

\section{Conclusions}

A recently proposed synthetic turbulent inlet condition for Large-Eddy Simulation (LES) has been used to predict the mean pressure and rootmean-squared (r.m.s.) of the pressure fluctuation over a CAARC building model surface. The new inlet condition has proven to be very successful in 
modelling the mean pressure. As for the r.m.s. of the pressure fluctuations, the present study has captured the detailed fluctuations on the corners of the CAARC building. Numerical experiments show that the inflow turbulence intensity has a significant effect on the surface pressure fluctuations, whereas the inflow turbulence integral length scales have a negligible effect, as long as they are within an appropriate range.

High correlations were observed between the surface pressures and the local wall-normal forces on the two lateral faces of the building. The dominant frequencies of the surface pressures, the local-wall normal forces and the wake lateral velocity are essentially identical, and in good agreement with that of the total lateral force. A conditional sampling and averaging technique was used to investigate the correlations of peak surface pressure on the two lateral faces and the lateral velocity component in the wake region. The peak surface pressure fluctuations were found (as expected) to be in anti-phase with those at the same height on the opposing lateral face, no doubt because of the antisymmetric nature of vortex shedding from the lateral faces. It was also found that there was a phase delay (of approximately $90^{\circ}$ ) between the negative peak of the triggering surface pressure and the negative peak lateral velocity (towards the triggering location) in the wake region near-the leeward face. This was associated with the travel time towards the wake region of the current vortex shed from the leading corner.

\section{Acknowledgements}

This project is supported by an EPSRC Case studentship and partly sponsored by Arup and Partners Ltd. We thank Drs Ender Ozkan, Ngai 
Yeung and Steven Downie of Ove Arup and Partners Ltd for their support throughout. SJD is grateful to Mr Yusik Kim for using the turbulence inflow generation functions in OpenFOAM. The computations were performed on the Iridis3 computational system, University of Southampton.

\section{References}

Antonia, R.A., 1981. Conditional sampling in turbulence measurement. Ann. Rev. Fluid Mech. 13, 131-56.

Bartoli, G., Ricciardelli, F., 2010. Characterisation of pressure fluctuations on the leeward and side faces of rectangular buildings and accuracy ofthe quasi-steady loads. J. Wind Eng. Ind. Aerodyn. 98, 512-9.

Bearman, P.W., Obasaju, E.D., 1982. An experimental study of pressure fluctuations on fixed and oscillating squaresection cylinders. J Fluid Mech. 119, 297-321.

Dagnew, A.K., Bitsuamlak, G.T., 2010. LES evaluation of wind pressures on a standard tall building with and without a neighboring building, in: In The Fifth International Symposium on Computational Wind Engineering: p. 23-27, Chapel Hill, North Carolina, USA.

Daniels, S.J., Castro, I.P., Xie, Z.T., 2012. The application of the divergencefree inlet condition on the caarc standard tall building model, in: 10th UK Conf. Wind Eng., Southampton, UK.

van Driest, E.R., 1956. On turbulent flow near a wall. AIAA 23, 1007-11. 
ESDU-85020, 2001. Characteristics of atmosphere turbulence near the ground, Part II: single point data for strong winds (neutral atmosphere). Technical Report 85020. Engineering Sciences Data Unit (ESDU).

Gioffre, G.M., Gusella, V., Grigoriu, M., 2001. Non-gaussian wind pressure on prismatic buildings. I: Stochastic field. J. Struct. Eng 127, 981-9.

Goliger, A.M., Milford, R.V., 1988. Sensitivity of the CAARC standard building model to geometric scale and turbulence. J. Wind Eng. Ind. Aerodyn. 31, 105-23.

Holdø, A.E., Houghton, E.L., Bhinder, F.S., 1982. Some effects due to variations in turbulence integral length scales on the pressure distribution on wind-tunnel models of low-rise buildings. J. Wind Eng. Ind. Aerodyn. 10, $103-15$.

Huang, P., Gu, M., 2005. Pressure and forces measurements on CAARC standard tall building in wind tunnel of Tong Ji university, in: In: 12th National Wind Engineering Conference of China.

Huang, S.H., Li, Q.S., Wu, J.R., 2010. A general inflow turbulence generator for large eddy simulation. J Wind Eng. Indust. Aerodyn. 98, 600-17.

Huang, S.H., Li, Q.S., Xu, S., 2007. Numerical evaluation of wind effects on a tall steel building by CFD. J Const. Steel Res. 63, 612-27.

Issa, R.I., 1985. Solution of the implicitly discretised fluid flow equations by operator-splitting. J. Compt. Phys. 62, 40-65. 
Kim, Y., Castro, I.P., Xie, Z.T., 2013. Inflow conditions for LargeEddy Simulations with incompressible flow solvers. Comput. Fluids (DOI:10.1016/j.compfluid.2013.06.001) in press.

Lam, K.M., Zhao, J.G., 2002. Occurrence of peak lifting actions on a large horizontal cantilevered roof. J. Wind Eng. Ind. Aerodyn. 90, 897-940.

Melbourne, W.H., 1980. Comparison of measurements on the CAARC standard tall building model in simulated model wind flows. J. Wind Eng. Ind. Aerodyn. 6, 73-88.

Moss, G.F., Wardlaw, R.L., 1970. A standard tall building model for comparison of simulated natural wind in wind tunnels. Technical Report C.C.662m Tech.25. C.A.A.R.C.

Obasaju, E.D., 1992. Measurement of forces and base overturning moments on the CAARC tall building model in a simulated atmospheric boundary layer. J. Wind Eng. Ind. Aerodyn. 40, 103-26.

OpenFOAM, 2010. User Guide. Technical Report. OpenFOAM.

Smirnov, A., Shi, S., Celik, I., 2001. Random flow generation technique for large-eddy simulations and particle dynamics modelling. Fluids Eng. 123, 359-71.

Surry, D., Djakovich, G., 1995. Fluctuating pressures on models of tall buildings. J. Wind Eng. Ind. Aerodyn. 58, 81-112.

Tamura, T., Nozawa, K., Kondo, K., 2008. AIJ guide for numerical prediction of wind loads on buildings. J. Wind Eng. Ind. Aerodyn. 96, 1974-84. 
539 Xie, Z.T., Castro, I.P., 2008. Efficient generation of inflow conditions for large eddy simulation of street-scale flow. Flow Turb. Combust. 81, 449-70.

${ }_{541}$ Xie, Z.T., Castro, I.P., 2009. Large-Eddy Simulation for flow and dispersion ${ }_{542}$ in urban streets. Atmos. Environ. 43, 2174-85. 